\title{
Predictores clínicos de enfermedad de Alzheimer en pacientes con deterioro cognitivo leve
}

Clinical Predictors of progression to Alzheimer disease in patients with amnestic mild cognitive impairment

\section{Objetivo}

Evaluar la importancia predictiva de mediciones neurocognitivas para la progresión de deterioro cognitivo leve tipo amnésico (DCL) a enfermedad de Alzheimer (EA).

\section{Diseño y lugar}

Cohorte de un ensayo aleatorizado ${ }^{1}$ llevado a cabo en EEUU y Cánada (69 sitios) entre 1999 y 2004.

\section{Pacientes}

539 pacientes de 55 a 90 años con resultados del "Minimental State Examination" mayor o igual a 24, "Dementia clinical scale" 0,$5 ; y$ sin enfermedad cerebrovascular significativa, enfermedades médicas o psiquiátricas que pudieran interferir con el estudio.

\section{Evaluación de factores pronósticos}

Fueron realizadas 11 mediciones neurocognitivas y tres escalas de severidad de demencia y de funcionalidad; y fueron recabados factores de riesgos demográficos (edad, sexo, educación) y genéticos (APO E) para calcular la probabilidad que un paciente con DCL desarrolle EA en un período de tres años.

\section{Medición de resultados principales \\ Desarrollo de EA probable o posible.}

\section{Resultados principales}

De los 539 participantes del estudio, 212 progresaron a EA, con una tasa de progresión del $16 \%$ anual. El modelo de predicción ajustado que incluyó APOE y las pruebas neurocognitivas "Symbol Digit Modalities Test", "New York University Paragraph Recall Test", "Alzheimer's Disease Assessment Scale-Cognitive subscale (ADAS-cog) total score" y el "Delayed
10-Word List Recall", tuvo una predicción 81\% (IC95\% 0,79 a 0,83 ) con una capacidad predictiva similar luego de excluir el resultado de APOE. La edad, el nivel educativo y el sexo fueron predictores independientes de progresión a EA. Ver tabla 1.

Tabla 1: predictores de la progresión a enfermedad de Alzheimer de pacientes con deterioro cognitivo leve.

\begin{tabular}{|c|c|c|}
\hline & $\begin{array}{c}\text { Precisión de cada } \\
\text { prueba en el análisis } \\
\text { univariable (\%) }\end{array}$ & IC95\% \\
\hline APO E4 & $64,1 \%$ & 0,641 a $\quad 0,655$ \\
\hline Synbol Digit Modalities Test & $70,7 \%$ & 0,699 a 0,717 \\
\hline New York University Paragraph Recall Test & $73,2 \%$ & 0,718 a 0,739 \\
\hline $\begin{array}{l}\text { Alzheimer's Disease Assessment Scale- } \\
\text { Cognitive subscale (ADAS-cog) total score }\end{array}$ & $73,7 \%$ & 0,73 a 0,747 \\
\hline Delayed 10-Word List Recall & $73,5 \%$ & 0,73 a 0,744 \\
\hline Las cuatro pruebas neurocognitivas & $80 \%$ & 0,78 a 0,82 \\
\hline Las cuatro pruebas neurocognitivas más APO E4 & $81 \%$ & 0,79 a 0,83 \\
\hline
\end{tabular}

ADAS-cog: Alzheimer's Disease Assessment Scale-Cognitive subscale total score.

\section{Conclusiones}

La progresión de DCL a EA en la cohorte evaluada pudo ser determinada con mayor exactitud utilizando una combinación de cuatro test cogntivos.

Palabras clave: daño cognitivo leve tipo amnésico, enfermedad de Alzheimer, test cognitivos, pronóstico.

Key words: Alzheimer disease, amnestic mild cognitive impairment, cognitive testing, prognosis.

Fuente de financiamiento: National Institute on Aging (UO1 AG10483), The Institute for the Study of Aging, Pfizer Inc., and Elisai Inc.

\section{Comentario}

Los déficits neurocognitivos que mejor predicen la evolución de DCL a EA no son claramente conocidos. La mayoría de los estudios se habían enfocado en una sola de las variables, ya sea demográficas como sexo, edad, pruebas neurocognitivas, o factores biológicos como la presencia de APOE ${ }^{1}$.

Teniendo en cuenta su relevancia y prevalencia en la población adulta mayor, es muy importante la búsqueda de información útil para predecir clínicamente que pacientes pueden evolucionar desde DCL a EA. El presente estudio fue realizado sobre una muestra de pacientes muy seleccionados por los criterios de exclusión, lo que puede implicar que no sean representativos de los que vemos en los consultorios de atención primaria. Su ventaja es que las pruebas neurocognitivas seleccionadas son breves y sencillas. Respecto de la medición de APOE y como informan los resultados de este estudio, su utilidad tiene reportes conflictivos en la literatura².

Los pacientes con DCL pueden tener la memoria afectada de forma similar a los pacientes con EA leve, pero estos últimos tienen otros dominios cognitivos afectados, pudiendo o nó estar correlacionados con los niveles de APOE. Hacen falta más estudios para determinar en que subgrupo de pacientes se justifica su medición.

\section{Conclusiones del comentador}

Si bien es importante tener instrumentos de medición que evalúen la progresión de DCL a EA, no está demostrado que las medicaciones utilizadas hoy en día para el tratamiento de los pacientes con demencia (inhibidores de la colinesterasa y vitamina E) sean útiles para reducir este riesgo de progresión3. Este es el aspecto que debe ser tenido en cuenta y evaluado en el futuro, lo que permitirá probar nuevas drogas. Por el momento, los expertos no recomiendan la evaluación de APOE en pacientes con DCL, lo que resulta confirmado por los resultados del presente estudio.

Javier Benchimol [ Servicio de Clínica Médica, Programa de Medicina Geriátrica del Hospital Italiano de Buenos Aires. Javier.benchimol@hospitalitaliano.org.ar ]

Benchimol J. Predictores clínicos de enfermedad de Alzheimer en pacientes con deterioro cognitivo leve. Evid Act Pract Ambul. 11(3):76. May-Jun 2008. Comentado de: Fleischer A y col. Clinical Predictors of progression to Alzheimer disease in amnestic mild cognitive impairment. Neurology. 2007; 68:1588-1595. PMID: 17287448.

Referencia

1. Petersen R y col. Mild Cognitive Impairment. Arch Neurol 1999; 56:303-308.

2. Tierney M y col. Neuropsychological tests accurately predict incident Alzheimer disease after 5 and 10 years. Neurology 2005; 64:1853-1859.

3. Petersen R y col. Vitamin E and Donepezil for the Treatment of Mild Cognitive Impairment. N Engl J Med 2005; 352:2379-2388. 